\title{
Reliability and validity of Simplified Chinese version of the Italian Spine Youth Quality of Life questionnaire in adolescents with idiopathic scoliosis
}

\section{Shanshan Liu}

Peking University Third Hospital

Junyang Liang

Weihai Wei People's Hospital

Nanfang Xu ( $\nabla$ xunanfang@foxmail.com)

Peking University Third Hospital https://orcid.org/0000-0001-5888-293X

\section{Shuo Mai}

Peking University Third Hospital

\section{Qi Wang}

Peking University Health Science Center

\section{Lin Zeng}

Peking University Third Hospital

\section{Chaojun Du}

Peking University Third Hospital

\section{Yaoxu Du}

Peking University Third Hospital

\section{Yan Zeng}

Peking University Third Hospital

\section{Miao Yu}

Peking University Third Hospital

Zhongjun Liu

Peking University Third Hospital

\section{Research}

Keywords: Quality of life, Adolescent idiopathic scoliosis, Cultural adaptation, Italian Spine Youth Quality of Life 
DOI: https://doi.org/10.21203/rs.3.rs-29311/v1

License: (c) (1) This work is licensed under a Creative Commons Attribution 4.0 International License. Read Full License 


\section{Reliability and validity of Simplified Chinese version of the Italian Spine}

\section{Youth Quality of Life questionnaire in adolescents with idiopathic scoliosis}

Shanshan Liu ${ }^{1 *}$, Junyang Liang ${ }^{2 *}$, Nanfang $\mathrm{Xu}^{1}$, Shuo Mai ${ }^{1}$, Qi Wang ${ }^{3}$, Lin Zeng ${ }^{4}$, Chaojun $\mathrm{Du}^{5}$, Yaoxu Du ${ }^{5}$, Yan Zeng ${ }^{1}$, Miao Yu' ${ }^{1}$, Zhongjun Liu ${ }^{1}$

1 Department of Orthopaedic Surgery, Peking University Third Hospital, Beijing, China

2 Department of Spinal Surgery, Weihai Wei People's Hospital, Weihai, China

3 Peking University Health Science Center, Beijing, China

4 Research center of clinical epidemiology, Peking University Third Hospital, Beijing, China

5 Department of Orthotics and Prosthetics, Peking University Third Hospital, Beijing, China

* These authors contributed to the work equally and should be regarded as co-first authors.

E-mail

Shanshan Liu: 1039165305@qq.com

Junyang Liang:whwjzwk@163.com

Nanfang Xu: xunanfang@foxmail

Shuo Mai: maishuo@bjmu.edu.com

Qi Wang: jodiwang0816@gmail.com

Lin Zeng: zlwhy@163.com

Chaojun Du: 838935061@qq.com

Yaoxu Du: 821017319@qq.com

Yan Zeng: puthzengyan@163.com

Miao Yu: puthyumiao@163.com

Zhongjun Liu: zjl@bjmu.edu.com

Corresponding author

Nanfang Xu, MD

Department of Orthopaedic Surgery

Peking University Third Hospital

Beijing 100191, China

Tel: +86-10-82267011

Fax: +86-10-82267368 


\section{Abstract}

Background: The Italian Spine Youth Quality of Life (ISYQOL) questionnaire is a tool used to evaluate health-related quality of life in adolescents with Idiopathic Scoliosis. The study aimed to undertake the process of cultural adaptation of the ISYQOL questionnaire into Simplified Chinese.

Methods: The translation from Italian into Simplified Chinese was performed. It involved 138 adolescents whose Cobb angle ranged between 20-40 degrees, 50 wearing the brace and 88 not wearing the brace. Statistical analysis calculated the reliability, floor and ceiling effects of the ISYQOL. After that, construct validity was measured by analyzing the ISYQOL relationship Scoliosis Research Society-22 patient Questionnaire (SRS-22).

Results: There were no floor or ceiling effects in ISYQOL questionnaire. Cronbach's alpha coefficient evaluated for Internal consistency was 0.75 without the brace and 0.88 with the brace. Intraclass correlation coefficients assessed with the use of the test-retest method was 0.72 without the brace and 0.80 with the brace. A strong relationship exists between the ISYQOL measure and SRS-22 scores (rho=0.63; $p<0.01$ ), reflecting the high validity of the questionnaires. Both ISYQOL and SRS-22 scores showed no statistical difference between groups with and without the brace $(p>0.05)$.

Conclusions: Trans-cultural validation in Chinses language showed the reliability and validity of the ISYQOL

Keywords: Quality of life, Adolescent idiopathic scoliosis, Cultural adaptation, Italian Spine Youth Quality of Life 


\section{Background}

Adolescent Idiopathic Scoliosis (AIS) is a frequent pathology among adolescents, the prevalence of which is 1-2\% [1]. Scoliosis itself and its treatment may have a severe impact on patients' health-related quality of life (HRQOL), although few patients are associated with severe complications.[2]. The need for a specific HRQOL measure in AIS has been recognized for a long time, which seems as important as radiologic measurements in evaluating treatment effect.[3] As a way of measuring HRQOL, self-administered questionnaires have become the most commonly used means to assess the patients' perspective of health. In 2017 Caronni et al. [4]proposed the ISYQOL questionnaire, showed high measurement properties in Rasch analysis. Further research has found this questionnaire of adolescents with spinal deformities better than SRS-22 questionnaire[5], which seems to be the most widely used as reference standard in the past[6]. ISYQOL was developed and validated in the Italian language. In order to be used in a different language speaking population, it has to be subject to a process called trans-cultural validation[7]. Reliability and validity of self-administered questionnaires must be evaluated In order to make it suitable for research and clinical practice[8]. Simplified Chinese is the contemporary Chinese primarily used in mainland China, Singapore, and Malaysia. The study was aimed to evaluate the reliability and validity of Simplified Chinese version of the ISYQOL (SC-ISYQOL) in adolescents with idiopathic scoliosis.

\section{Methods}

\section{Questionnaire}

ISYQOL Questionnaire is a 20 Likert scale items questionnaire and consisted of two domains (13 items of the spine health and 7 of the brace domains). The first version was designed to be administered to patients wearing the brace and consisted of both the spine health and brace domains (full questionnaire). The second version was designed to be administered to patients not wearing the brace and consisted of the spine health domain only. Scoring of the ISYQOL was planned as follows: Items investigating the presence of spine-related problems are coded 0,1 , or 2 ( 0 , never; 1 , sometimes; 2 , often). Items investigating the presence of positive thoughts are coded 2, 1 , or 0 (2, never; 1 , sometimes; 0 , often). The ISYQOL total score is obtained by adding up all single items, and the lower the category, the better quality of life. Using Rasch analysis, the ordinal ISYQOL total score is converted to an interval measure (i.e. ISYQOL measure), which is expressed on a 0\%-100\% scale (with 100\% indicating great quality of life). ISYQOL measure can be used to compare the HRQOL of patients with or without the brace, who filled in different versions of the questionnaire. SRS-22 comprises 5 domains (Function, Pain, Mental Health, Self-Image, Management Satisfaction/Dissatisfaction) up to a total of 22 items. The score ranges from 1 to 5 points for each item, with a summary score between 22 and 110[6]. Higher scores are better quality of life. In our study, we used Simplified Chinese validated version of SRS-22 (SC-SRS-22)[9, 10].

\section{Translation}

We followed widely accepted guidelines described by Guillemin for translation and crosscultural adaptation of ISYQOL questionnaires[6, 7]. In the first stage, two independent 
translators converted the original Italian text into Simplified Chinese. One of the translators, who had a medical background, was instructed on the whole process of adaptation. The other translator had no medical background and received no information on the project. The second stage consisted of a comparison of the original and two translated versions. During that stage, the two translators and the authors identified differences in translations and produced a combined version. In the third stage - the so-called reversed translationtwo independent translators, who were native in Italy, translated the Simplified Chinese version into the language of the original document (Italian). The translators were not familiar with the original version. The objective of this stage was to assure the equivalence of the two versions and to identify possible mistranslations. At the last fourth stage, a commission composed of a specialist in orthopedics, translators, a statistician, and a psychologist reviewed the translations. As a result of consensus, the so-called pre-final version SC-ISYQOL was drafted.

\section{Sample}

The study took place from February 2019 to November 2019 with approval of the local Hospital Ethics Committee. After obtaining patients and their guardians ' consent, we administered the SC-ISYQOL and SC-SRS-22 questionnaire to Chinese adolescents age 1018 years with idiopathic scoliosis at the end of the clinical visit. The study included 138 consecutive patients whose Cobb angle ranged between 20-40 degrees and without a history of spine surgery. A dedicated research assistant was responsible for ascertaining the on-site completion of all questionnaire items by the participants during their office visits. We randomly selected 70 patients for the test-retest, with 12-16 days in interval. Among them, fifty patients wearing the brace who were treated with the same kind of brace for at least 4 months, at least $10 \mathrm{~h}$ per day filled out the full questionnaire, while 88 patients not wearing the brace filled out the spine health domain only.

Statistical analysis

Statistical analysis was performed using Statistics 26.0 software. Firstly, descriptive statistics were used to calculate mean scores and standard deviations for a given question and a domain. The second level was comparative, concerning reliability and validity. The two most important properties of reliability are consistency and stability. Internal consistency was assessed using Cronbach' $s$ alpha coefficient. Test-retest design was used to measure the temporal stability of the questionnaire with intraclass correlation coefficient (ICC). Construct validity assessed by comparing ISYQOL measure with SRS-22 through Spearman rank correlation coefficient $(r)$. The independent sample $t$ test was used to compare the differences in quality of life scores between the two groups with or without braces. For all tests, statistical significance was set at $p<0.05$.

\section{Results}

The sample included 113 girls and 25 boys, whose mean age at the time of questionnaire administration was 13.7 years ( \pm 2.3 years). Because patients with or without braces filled out different versions of the ISYQOL questionnaire, they were divided into two groups, brace group and no brace group. The Cobb angle ranged between 21.1-38.5 degrees, and for demographic and clinical characteristics details, see Table 1. 
There are 16 (0.6\%) missing values in all questionnaires, and we use mean to fill in missing values in this study. A dedicated research assistant ensures better questionnaire quality. Mean, median, minimal, maximal score, standard deviation and interquartile range of ISYQOL are presented in Table 2. The mean score for ISYQOL was 8.5 (ranged between 1-20) without the brace and 14.2 (ranged between 2-33) with the brace. Because first version has more questions than second version, the scores of the two groups are not comparable. There are no floor and ceiling effects in ISYQOL questionnaire in both versions.

Mean, standard deviation, number of options for each ISYQOL question are presented in Table 3. Mean values for individual ISYQOL items ranged from 0.3 (Item 6 and 13) to 1.1 (Item 1, 9 and 20). More than 70\% of patients selected "often" in Item 6 and 13. They are "Despite your back problem, is your life normal?" and "Despite your back problem, do you live a happy life?"

The value of Cronbach' $s$ alpha is presented in Table 4. In no brace version, Cronbach' $s \alpha$ coefficient was 0.75 in the spine health domain. In brace version, Cronbach' $s$ a coefficient was 0.88 , with 0.85 in spine health domain and 0.86 in brace domain. All of them indicated excellent internal consistency reliability.

Fifty percent of patients or more retested both versions of the questionnaire. ICC assessed was 0.72 in no brace version and 0.80 in brace version. Both versions indicated excellent temporal stability[11].

ISYQOL measure is possible to compare patients not wearing the brace (who fill 13 out of 20 items only) with wearing the brace (who fill the full questionnaire). Mean, standard deviation for ISYQOL measure and SRS22 scores are presented in Table 5. The Spearman rho between ISYQOL measure and SRS-22 scores was 0.63 with highly significative value $(p<0.001)$ Moreover, we used the ISYQOL measure $(p=0.425)$ and SRS-22 scores $(p=0.229)$ to compare the difference in HRQOL between brace group and no brace group. Both scores showed no statistical difference between groups with and without braces.

\section{Discussion}

HRQOL in patients with a spine deformity can be decreased for several reasons, especially in adolescents[12, 13]. For example, AIS can lead to physical, psychological, and social impairments that eventually have an impact on HRQOL[12]. Besides, conservative treatment of scoliosis with a rigid brace can be negative to affect their QOL. [14]

In this study, patients wearing the brace would take $X$-rays with and without the brace. We measured Cobb angle from $X$-rays without the brace, and strictly chose patients with Cobb angle ranging between 20-40 degrees, making the sample included in the study fitted the indications for braces for AIS. When evaluating the effect of brace treatment, not only the changes in the degree of scoliosis in radiologic measurements, but also the changes in QOL must be considered[14]. In turn, QOL is closely related to patients' compliance with brace treatment and depends on the conservative treatment effect[15, 16].

ISYQOL questionnaire was designed to evaluate the HRQOL of adolescents with idiopathic scoliosis. Some papers perceived the need to use disease specific questionnaires Instead of generic questionnaire[17-19]. From the total questionnaire score, $0 \%$ of patients scored at floor and 0\% scored at ceiling, showing no floor and ceiling effects. 
Cronbach' s alpha is the most commonly applied statistical parameter for showing the internal consistency of an instrument [20]. The SC-ISYQOL had a high value of Cronbach' s alpha coefficient (0.88 and 0.75), whether wearing brace or not, exceeding the minimum recommended value of 0.70 and indicating satisfactory internal consistency as a factor of satisfactory reliability of the SC-ISYQOL.

Intraclass correlation coefficients (ICC) of the SC-ISYQOL assessed with the use of testretest method was 0.72 without brace and 0.80 with brace, showing a good temporal stability.

There are some questions designed for wearing the brace in ISYQOL questionnaire. ISYQOL measure can convert scores of with and without the brace to standard measurement, so that is used to compare the total score of the two versions. It is good for evaluating the changes of HRQOL before and after brace treatment.

Criterion validity is the correlation of a scale with a valid, accepted universally acknowledged measure of the trait or disorder under study. The outcome measures for construct validity were adopted SRS-22, most widely used as reference standard in the past, in order to evaluate the relationship with another patient-oriented questionnaire not focused on brace therapy. The results of the current study showed that a strong relationship exists between the ISYQOL measure and SRS-22 scores (rho=0.63; $p<0.01$ ), reflecting the high validity of the questionnaires. This relationship was also found in the study by Caronni al.[5], with rho= 0.71 and $p<0.01$.

There is no difference in HRQOL between brace group and no brace group, whether through ISYQOL or SRS-22. Earlier reports reported that braces have impacted HRQOL may due to factors such as stiffness of brace[14], but it did not appear in this study. This may be related to the 3D printing brace applied in the hospital. This brace fits the patient's body design and is custom-made by 3D printing, having good fit and comfort. However, this is a cross-sectional study, with different sample of the 2 groups. This view needs to be followed up and compared by the same person's QOL score before and after brace treatment. In our study, there are limitations such as cross-sectional design and the single clinical center available in the cross-cultural adaptation process. The ISYQOL questionnaire needs to be promoted and applied in clinical and research, and multi-center cooperation has been obtained for further confirmation.

\section{Conclusion}

To conclude, the culturally adapted Chinese version of the ISYQOL showed excellent reliability, high internal consistency and satisfactory concurrent validity. This instrument is therefore useful as a clinical evaluation tool for Chinese scoliosis patients. 
Table 1 Participants' demographics and clinical data

\begin{tabular}{llll}
\hline & No brace & Brace & Total \\
\hline $\mathrm{N}$ & 88 & 50 & 138 \\
Males vs. females, $\mathrm{N}$ & 21 vs 67 & 4 vs 46 & 25 vs 113 \\
Mean age (SD), years & $13.6(2.3)$ & $13.9(2.3)$ & $13.7(2.3)$ \\
Mean body weight (SD), kg & $47.5(12.9)$ & $46.4(9.0)$ & $47.1(11.7)$ \\
Mean height (SD), cm & $160.3(11.5)$ & $160.5(13.5)$ & $160.4(12.2)$ \\
Cobb angle, Min-Max & $21.1-38.5$ & $26.9-33.7$ & $21.1-38.5$ \\
Test-retest, N & 44 & 26 & 70 \\
\hline
\end{tabular}

N: number of participants; SD: standard deviation; Min: minimal; Max: maximal

Table 2 Mean, median, minimal, maximal score, standard deviation and interquartile range of ISYQOL

\begin{tabular}{lllllll}
\hline Questionnaire & Mean & SD & Min & Max & Median & IQR \\
\hline No brace & 8.5 & 4.2 & 1 & 20 & 9 & 6 \\
Brace & 14.2 & 7.4 & 2 & 33 & 13.5 & 11 \\
\hline
\end{tabular}

SD: standard deviation; Min: minimal; Max: maximal; IQR: interquartile range

Table 3 Mean, standard deviation, number of options for each ISYQOL item

\begin{tabular}{|c|c|c|c|c|c|c|c|c|c|c|}
\hline \multirow{3}{*}{ Item } & \multicolumn{5}{|c|}{ No brace } & \multicolumn{5}{|c|}{ Brace } \\
\hline & \multirow[t]{2}{*}{ Mean } & \multirow[t]{2}{*}{ SD } & \multicolumn{3}{|c|}{ number\% } & \multirow[t]{2}{*}{ Mean } & \multirow[t]{2}{*}{ SD } & \multicolumn{3}{|c|}{ number\% } \\
\hline & & & 0 & 1 & 2 & & & 0 & 1 & 2 \\
\hline 1 & 1.1 & 0.6 & 11 & 68 & 20 & 1.1 & 0.5 & 8 & 74 & 18 \\
\hline 2 & 0.8 & 0.6 & 30 & 59 & 11 & 0.5 & 0.6 & 54 & 40 & 6 \\
\hline 3 & 0.6 & 0.6 & 50 & 43 & 7 & 0.6 & 0.7 & 52 & 38 & 10 \\
\hline 4 & 0.9 & 0.6 & 26 & 63 & 11 & 0.8 & 0.7 & 34 & 50 & 16 \\
\hline 5 & 0.5 & 0.7 & 59 & 31 & 10 & 0.6 & 0.7 & 54 & 34 & 12 \\
\hline 6 & 0.4 & 0.6 & 74 & 17 & 9 & 0.3 & 0.6 & 72 & 22 & 6 \\
\hline 7 & 0.5 & 0.6 & 55 & 40 & 6 & 0.5 & 0.6 & 54 & 40 & 6 \\
\hline 8 & 0.8 & 0.7 & 34 & 50 & 16 & 0.8 & 0.6 & 26 & 66 & 8 \\
\hline 9 & 1.1 & 0.6 & 18 & 58 & 24 & 1.1 & 0.6 & 12 & 64 & 24 \\
\hline 10 & 1.0 & 0.6 & 22 & 61 & 17 & 0.8 & 0.7 & 32 & 52 & 16 \\
\hline 11 & 0.6 & 0.8 & 56 & 28 & 16 & 0.6 & 0.8 & 60 & 24 & 16 \\
\hline 12 & 0.8 & 0.8 & 39 & 41 & 20 & 0.9 & 0.8 & 42 & 30 & 28 \\
\hline 13 & 0.4 & 0.6 & 66 & 27 & 7 & 0.3 & 0.6 & 76 & 16 & 8 \\
\hline 14 & & & & & & 0.7 & 0.7 & 46 & 38 & 16 \\
\hline 15 & & & & & & 0.9 & 0.8 & 34 & 40 & 26 \\
\hline 16 & & & & & & 0.8 & 0.6 & 30 & 62 & 8 \\
\hline 17 & & & & & & 0.8 & 0.7 & 34 & 50 & 16 \\
\hline 18 & & & & & & 0.4 & 0.6 & 64 & 28 & 8 \\
\hline 19 & & & & & & 0.4 & 0.6 & 64 & 28 & 8 \\
\hline 20 & & & & & & 1.1 & 0.7 & 16 & 56 & 28 \\
\hline
\end{tabular}


Table 4 The value of Cronbach' s alpha

\begin{tabular}{ll}
\hline Questionnaire & $\begin{array}{l}\text { Cronbach' s } \\
\text { alpha }\end{array}$ \\
\hline No brace & 0.75 \\
Brace & 0.88 \\
$\quad$ spine health domain & 0.85 \\
brace domain & 0.86 \\
\hline
\end{tabular}

Table 5 The mean and standard deviation for ISYQOL measure and SRS22 scores

\begin{tabular}{lll}
\hline & ISYQOL measure (\%) & SRS22 scores \\
\hline Total & $56.7(11.0)$ & $90.9(9.3)$ \\
No Brace & $56.2(11.0)$ & $91.6(8.5)$ \\
Brace & $57.7(11.1)$ & $89.6(10.5)$ \\
\hline
\end{tabular}

Mean (Standard deviation) 


\section{List of abbreviations}

$\begin{array}{ll}\text { ISYQOL } & \text { Italian Spine Youth Quality of Life } \\ \text { SRS-22 } & \text { Scoliosis Research Society-22 patient Questionnaire } \\ \text { AIS } & \text { Adolescent Idiopathic Scoliosis } \\ \text { HRQOL } & \text { Health-related quality of life } \\ \text { SC-ISYQOL } & \text { Simplified Chinese version of the ISYQOL } \\ \text { SC-SRS-22 } & \text { Simplified Chinese validated version of SRS-22 } \\ \text { ICC } & \text { Intraclass correlation coefficient }\end{array}$

\section{Declarations}

\section{Ethics approval and consent to participate}

Permission to perform the study was obtained from the Clinical Research Ethics Committee of the Peking University Third Hosiptal.

\section{Consent for publication}

Not applicable.

\section{Availability of data and materials}

Data can be shared upon contact with the correspondence author.

\section{Competing interests}

The authors declare that they have no competing interests.

\section{Funding}

This work was supported by grants from the Young Scientists Fund of the National Natural Science Foundation of China (CN) (No. 2019NSFC81901822), Peking University Fund of Fostering Young Scholars' Scientific \& Technological Innovation (No. BMU2018PYB016) and Capital's Fund for Health Development and Research (No. 2018-4-4097).

\section{Authors' contributions}

Shanshan Liu, Junyang Liang, Nanfang Xu, Miao Yu, Yan Zeng and Zhongjun Liu participated in the conception and design of the study. Nanfang $X u$, Shuo Mai, Qi Wang, Chaojun Du and Yaoxu Du participated in the acquisition of data. Shanshan Liu, Junyang Liang, Nanfang Xu and Lin Zeng participated in the analysis and interpretation of data. Shanshan Liu and Junyang Liang wrote the draft of the manuscript. All authors critically revised the manuscript for important intellectual content and approved the final version to be published.

\section{Acknowledgements}

The authors wish to thank the patients who provided their time to participate in the study.

\section{Authors' information}

Not applicable. 
1. Willner S, Uden A: A prospective prevalence study of scoliosis in Southern Sweden. Acta Orthop Scand 1982, 53:233-237.

2. Tones M, Moss N, Polly DW, Jr:: A review of quality of life and psychosocial issues in scoliosis. Spine (Phila $P a$ 1976) 2006, 31:30273038.

3. Haher TR, Gorup JM, Shin TM, Homel P, Merola AA, Grogan DP, Pugh L, Lowe TG, Murray M: Results of the Scoliosis Research Society instrument for evaluation of surgical outcome in adolescent idiopathic scoliosis. A multicenter study of $\mathbf{2 4 4}$ patients. Spine (Phila Pa 1976) 1999, 24:14351440.

4. Caronni A, Sciume L, Donzelli S, Zaina F, Negrini S: ISYQOL: a Raschconsistent questionnaire for measuring health-related quality of life in adolescents with spinal deformities. Spine J 2017, 17:1364-1372.

5. Caronni A, Donzelli S, Zaina F, Negrini S: The Italian Spine Youth Quality of Life questionnaire measures health-related quality of life of adolescents with spinal deformities better than the reference standard, the Scoliosis Research Society 22 questionnaire. Clin Rehabil 2019, 33:1404-1415.

6. Asher M, Min Lai S, Burton D, Manna B: The reliability and concurrent validity of the scoliosis research society-22 patient questionnaire for idiopathic scoliosis. Spine (Phila Pa 1976) 2003, 28:63-69.

7. Guillemin F: Cross-cultural adaptation and validation of health status measures. Scand J Rheumatol 1995, 24:61-63.

8. Ware JE, Jr., Sherbourne CD: The MOS 36-item short-form health survey (SF-36). I. Conceptual framework and item selection. Med Care 1992, 30:473-483.

9. Li M, Wang CF, Gu SX, He SS, Zhu XD, Zhao YC, Zhang JT: Adapted simplified Chinese (mainland) version of Scoliosis Research Society-22 questionnaire. Spine (Phila Pa 1976) 2009, 34:1321-1324.

10. Qiu G, Qiu Y, Zhu Z, Liu Z, Song Y, Hai Y, Luo Z, Liu Z, Zhang H, Lv G, et al: Reevaluation of reliability and validity of simplified Chinese version of SRS-22 patient questionnaire: a multicenter study of 333 cases. Spine (Phila Pa 1976) 2011, 36:E545-550.

11. Mehta S, Bastero-Caballero RF, Sun Y, Zhu R, Murphy DK, Hardas B, Koch G: Performance of intraclass correlation coefficient (ICC) as a reliability index under various distributions in scale reliability studies. Stat Med 2018, 37:2734-2752.

12. Freidel K, Petermann F, Reichel D, Steiner A, Warschburger P, Weiss HR: Quality of life in women with idiopathic scoliosis. Spine (Phila Pa 1976) 2002, 27:E87-91.

13. Freidel K, Reichel D, Steiner A, Warschburger P, Petermann F, Weiss HR: Idiopathic scoliosis and quality of life. Stud Health Technol Inform 2002, 88:24-29. 
14. Aulisa AG, Guzzanti V, Perisano C, Marzetti E, Specchia A, Galli M, Giordano $\mathrm{M}$, Aulisa L: Determination of quality of life in adolescents with idiopathic scoliosis subjected to conservative treatment. Scoliosis 2010, 5:21.

15. Bunge EM, Habbema JD, de Koning HJ: A randomised controlled trial on the effectiveness of bracing patients with idiopathic scoliosis: failure to include patients and lessons to be learnt. Eur Spine J 2010, 19:747753.

16. Landauer F, Wimmer C, Behensky $\mathrm{H}$ : Estimating the final outcome of brace treatment for idiopathic thoracic scoliosis at 6-month follow-up. Pediatr Rehabil 2003, 6:201-207.

17. Aprile I, Ruggeri AE, Savi Scarponi F, Illiano S, Foschini M, Vergili G, Frasca G, Padua L: Health-related quality of life in patients with adolescent idiopathic scoliosis after treatment: short-term effects after brace or surgical treatment, a comment. Eur Spine J 2007, 16:1962-1963; author reply 1964 .

18. Padua R, Ceccarelli E, Aulisa AG, Pitta L, Aulisa L: Outcome of Harrington surgery for idiopathic scoliosis. SF-36 and Roland questionnaires assessment. Stud Health Technol Inform 2002, 88:404.

19. Padua R, Padua S, Aulisa L, Ceccarelli E, Padua L, Romanini E, Zanoli G, Campi A: Patient outcomes after Harrington instrumentation for idiopathic scoliosis: a 15- to 28-year evaluation. Spine (Phila Pa 1976) 2001, 26:1268-1273.

20. Feise RJ, Donaldson S, Crowther ER, Menke JM, Wright JG: Construction and validation of the scoliosis quality of life index in adolescent idiopathic scoliosis. Spine (Phila Pa 1976) 2005, 30:1310-1315. 